Measurements of Positions of Asteroids in 1909. By E. S. Manson, Jr. $12 \frac{1}{2}$-inch Equatorial and Filar Micrometer of the Emerson McMillin Observatory of the Ohio State University at Columbus.

\begin{tabular}{l|l|l|l|l|l|l|l|l|l|}
\hline 1909 & Greenw.M.T. & $\Delta \alpha$ & $\Delta \delta$ & Cp. & $\alpha$ app. & $\log p \cdot \Delta \mid$ & $\delta$ app. & $\log p \cdot \Delta \mid \operatorname{Red}$. ad l. app. & $*$ \\
\hline
\end{tabular}

(26) Proserpina.

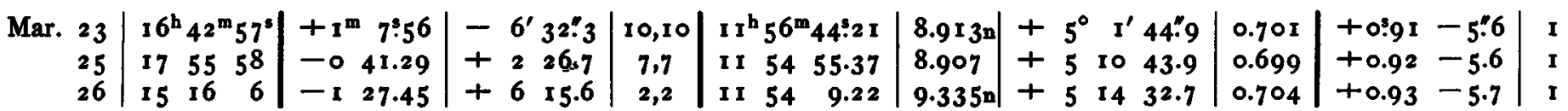

(532) Herculina.

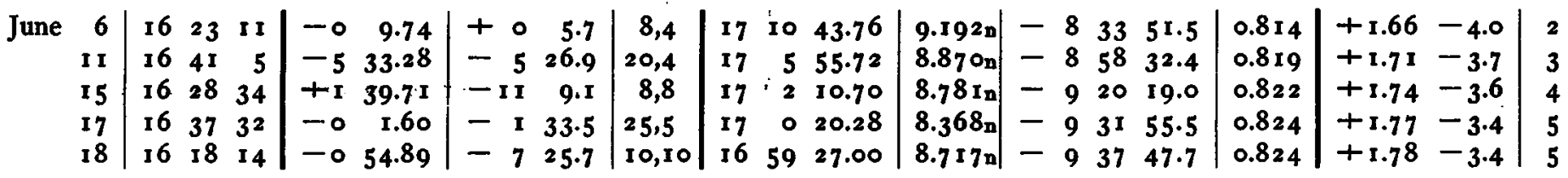

(444) Gyptis.

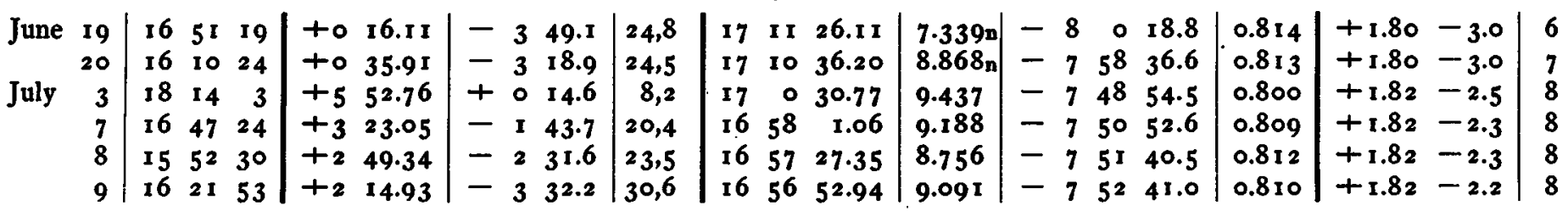

(19) Fortuna.

\begin{tabular}{ll|lll|ll|ll|l|lll|} 
July & 17 & 16 & 37 & 3 & -1 & 39.98 & +2 & 2.6 & 40,8 & 19 & 58 & 45.77
\end{tabular}

\begin{tabular}{l|lll|ll|ll|l|lll|}
18 & 17 & 30 & 50 & -2 & 42.05 & -0 & 43.0 & 25,5 & 19 & 57 & 43.72
\end{tabular}

$1917543^{8}-036.93+831.528,6 \quad$ J9 5642.54

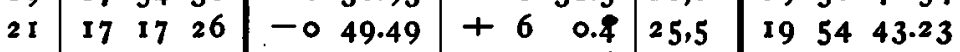

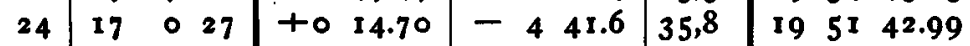

\begin{tabular}{lr|lll|lr|rr|rr|rr} 
Aug. 6 & 16 & 16 & 47 & -0 & 5.35 & -4 & 17.1 & 36,8 & 19 & 39 & 27.13
\end{tabular}

\begin{tabular}{|l|rrr|r|r|r}
$9.167 \mathrm{n}$ & -17 & 55 & 25.5 & 0.865 & $+2.24+4.6$ & $y$ \\
$8.443 \mathrm{n}$ & -17 & 58 & 11.1 & 0.871 & $+2.26+4.6$ & 9 \\
8.501 & -18 & 0 & 51.6 & 0.871 & $+2.27+4.4$ & 10 \\
$8.396 \mathrm{n}$ & -18 & 6 & 12.1 & 0.867 & $+2.28+4.3$ & 11 \\
$8.468 \mathrm{n}$ & -18 & 14 & 20.6 & 0.872 & $+2.29+3.8$ & 12 \\
8.056 & -18 & 48 & 50.2 & 0.875 & $+2.37+3.5$ & 13
\end{tabular}

(5II) Davida.

\begin{tabular}{|ll|lll|ll|lll|l|lll|l|l|lll|l|l|l} 
July & 19 & 16 & 23 & 16 & to & 49.24 & - & 0 & 8.7 & 19,4 & 17 & 42 & 18.40 & 9.075 & -17 & 51 & 25.8 & 0.867 & +2.08 & -2.2 & 14
\end{tabular}

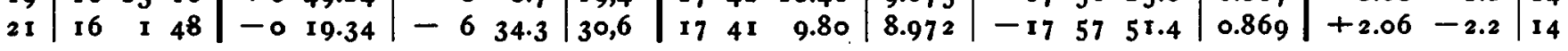

(16) Psyche.

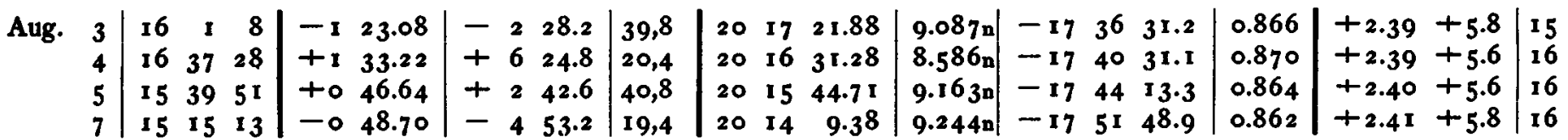

Mean Places of Comparison Stars.

\begin{tabular}{|c|c|c|c|}
\hline$*$ & $\alpha 1909.0$ & $\delta$ I909.0 & Authority \\
\hline & $\mathrm{II}^{\mathrm{h}} 55^{\mathrm{m}} 35^{\mathrm{s}} 74$ & $+5^{\circ} 8^{\prime} 22^{\prime \prime} 8$ & AG Lpz II 5985 \\
\hline 2 & $17 \quad 10 \quad 5^{1.84}$ & -83353.2 & AG Ott 5875 \\
\hline 3 & $17 \quad 1127.29$ & $\begin{array}{lll}-8 & 53 & 1.8\end{array}$ & $\begin{array}{l}1878 \\
\end{array}$ \\
\hline 4 & $17 \bigcirc 29.25$ & -99 & 5842 \\
\hline 5 & 17020.11 & -93018.6 & 5840 \\
\hline & 17 II 8.20 & $\begin{array}{llll}-7 & 56 & 26.7\end{array}$ & 5876 \\
\hline 8 & $17 \quad 958.49$ & - 75514.7 & 5870 \\
\hline & 165436.19 & -7496.6 & $5^{824}$ \\
\hline
\end{tabular}

\begin{tabular}{|c|c|c|c|}
\hline * & $\alpha 1909.0$ & d 1909.0 & Authority \\
\hline 9 & $20^{\mathrm{h}} \quad 0^{\mathrm{m}} 23^{8} 5^{\mathrm{I}}$ & $-17^{\circ} 57^{\prime} 32^{\prime \prime} 7$ & AG Wa 7548 \\
\hline ro & $1957 \quad 57.20$ & $-18 \quad 9 \quad 27.5$ & $A O ̈ 20171$ \\
\hline I 1 & $19 \quad 55 \quad 30.44$ & $\begin{array}{lll}-18 & 12 & 16.8\end{array}$ & $" 20150$ \\
\hline 12 & 195126.00 & $-18 \quad 9 \quad 42.8$ & $" 20087$ \\
\hline 13 & 193930.11 & $-18443^{6.6}$ & $\$ \quad 19918$ \\
\hline 14 & $\begin{array}{lll}17 & 41 & 27.08\end{array}$ & $\begin{array}{llll}-17 & 51 & 14.9\end{array}$ & AG Wa 6397 \\
\hline 15 & $20 \quad 18 \quad 42.57$ & $\begin{array}{lll}-17 & 34 & 8.8\end{array}$ & 7671 \\
\hline 16 & $\begin{array}{lll}20 & 14 & 55.67\end{array}$ & -1747 & $764 \mathrm{I}$ \\
\hline
\end{tabular}


Comparison of observed places with ephemerides.

\begin{tabular}{|c|c|c|c|c|c|c|c|c|c|c|c|}
\hline \multirow{4}{*}{$\begin{array}{c}\text { Planet } \\
\text { (26) Proserpina }\end{array}$} & \multirow{2}{*}{\multicolumn{2}{|c|}{ I909 }} & \multirow[b]{2}{*}{$\Delta \alpha$} & \multirow{2}{*}{$\Delta \delta$} & \multirow{3}{*}{ Ephem. } & \multirow{3}{*}{$\begin{array}{c}\text { Planet } \\
\text { (I }) \text { Fortuna }\end{array}$} & \multirow{2}{*}{\multicolumn{3}{|c|}{1909}} & \multirow[b]{2}{*}{$\Delta \delta$} & \multirow[b]{2}{*}{ Ephem. } \\
\hline & & & & & & & & & $\Delta \alpha$ & & \\
\hline & Mar. & 23 & $+3^{562}$ & -25.0 & & & July & 24 & -8.29 & -9.5 & \\
\hline & & 25 & +2.87 & -21.6 & & & Aug. & 6 & -7.93 & & \\
\hline & & 26 & +3.13 & --18.3 & & (r6) Psyche & Aug. & 3 & $-6 \mathrm{I} .2$ & $-I_{7} \mathrm{I}$ & Eph. comp. \\
\hline (19) Fortuna & July & $\mathrm{I} 7$ & -8.18 & - I 1.8 & B. J. I I II & & & 4 & $-6 \mathrm{r}: 4$ & -172 & from B. J. I I I I \\
\hline & & I 8 & $-8 . \mathrm{I} \mathrm{I}$ & -12.2 & & & & 5 & $-6 \mathrm{I} . \mathrm{I}$ & -172 & \\
\hline & & I 9 & $-8 . \times 6$ & -9.5 & & & & 7 & --61.1 & $-\mathrm{I} 7 \mathrm{I}$ & \\
\hline
\end{tabular}

Columbus, Ohio, 19 I F February 23.

E. S. Manson.

\section{Beobachtungen der Anhaltsterne des Winneckeschen Kometen (A. N. 4420) mit dem Meridiankreise der Sternwarte Santiago de Chile. Von F. W. Ristenpart.}

In den Beobachtungen des Winneckeschen Kometen I 909 d, die A. N. 4420 mitgeteilt sind, und zwar während meiner Abwesenheit durch den Chef der Äquatorial-Sektion Herrn Dr. Richard Prager, findet sich bei den Anschlüssen des 3. November eine Diskrepanz zwischen der auf dem GZStern und der auf dem Stern des Neuen Cordoba-GeneralKatalogs beruhenden Position in RA. von $3^{\mathrm{s} .07}$ für $\mathrm{Ri}, 2^{\mathrm{s}} .89$ für $\mathrm{Pr}$, die eine Untersuchung verdiente. Da eine Revision der Beobachtung nichts $\mathrm{zu}$ Tage förderte, was $\mathrm{zu} Z_{\text {weifeln }}$ Anlaß geben konnte, so konnte an eine starke E. B. eines der beiden Sterne gedacht werden. Mit Rücksicht darauf, daß bei der Mehrzahl der Anhaltsterne die Epoche bereits 35 Jahre zurücklag, und um zugleich der ganzen Reihe eine größere Homogenität zu geben, entschloß ich mich daher, sämtliche für den Winneckeschen Kometen in Santiago benutzten Anhaltsterne am Eichensschen Meridiankreise von I $7 \mathrm{~cm}$ Öffnung neu bestimmen zu lassen und zwar zweimal in jeder Lage des. Instruments, was auch

I 9 Io Aug. 5 Schraube Ost durch Ristenpart und Prager

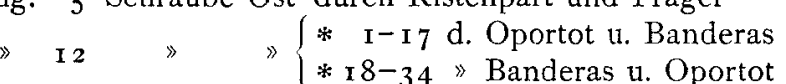

I 9 I0 Aug. 2 I Schraube West durch Ristenpart und Prager " 28 " " 8 Soza und Oportot

geschah. Die nachstehend als Mittel aus diesen 4 Abenden mitgeteilten Positionen gehören also zu der Epoche I9 10.64.

Als Anhaltsterne dienten 17 Sterne zwischen $-2 \mathbf{I}^{\circ}$ und $-38^{\circ}$ Deklination der Washingtoner Sternephemeriden, welche nicht nur wegen der Reichhaltigkeit an südlichen Sternen, sondern auch wegen der geringen Meridiandifferenz für Santiago sehr bequem sind. Es ergab sich als mittlerer Fehler einer Sternposition, ohne zwischen den Beobachtern zu unterscheiden, \pm 0.059 in RA. und \pm 0.90 in Dekl., so daß die auf vier Einzelpositionen beruhenden Mittelwerte die mittleren Fehler resp. $\pm 0.03 \circ$ und \pm 0.45 haben. Die für das mittlere $\ddot{A}$ quinoktium I 9 I o erhaltenen Positionen werden nachstehend einmal für das mittlere Äquinoktium I909 auf welches auch die Beobachtungen des Kometen r gog d bezogen sind, und dann auf das Normal-Äquinoktium 1925 bezogen mitgeteilt. Auf letzteres sind sie mit den Reduktionstafeln gebracht worden, welche als Publikation Nr. I der Sternwarte Santiago soeben die Presse verlassen.

\begin{tabular}{|c|c|c|c|c|c|c|c|c|c|c|c|c|}
\hline \multirow{2}{*}{$\begin{array}{l}\mathrm{Nr} . \\
\mathrm{I}\end{array}$} & \multirow{2}{*}{$\begin{array}{l}\begin{array}{c}\text { Bezeichnung des Sterns } \\
\text { in A. N. } 44^{20}\end{array} \\
\text { GZ } 17^{\mathrm{h}} \times 337\end{array}$} & \multicolumn{2}{|r|}{$\alpha 1909.0$} & \multicolumn{2}{|c|}{$\delta 1909.0$} & \multicolumn{2}{|r|}{$\alpha_{1925.0}$} & \multicolumn{3}{|c|}{81925.0} & \multicolumn{2}{|c|}{$\begin{array}{c}\text { Differenz } \\
\text { Santiago-Kataloge } \\
\Delta \alpha \quad \Delta \varepsilon \\
\end{array}$} \\
\hline & & I $7^{\mathrm{h}}$ & $2 I^{\mathrm{m}} 5^{6.26}$ & $-28^{\circ}$ & $6^{\prime} \quad 7^{\prime \prime} \cdot 3$ & I $7^{1}$ & $22^{m} 5^{6 s} 69$ & $-28^{\circ}$ & $6^{\prime}$ & 59.5 & +0.02 & $-1: 3$ \\
\hline 2 & $\mathrm{GZ}$ I $7^{\mathrm{h}}$ I 347 & & $22 \quad 5.87$ & -28 & 530.8 & I 7 & 236.30 & -28 & 6 & 22.9 & -0.34 & $-4 \cdot 3$ \\
\hline 3 & $\mathrm{GZ}$ I $7^{\mathrm{h}}$ I $50 \mathrm{I}$ & & 24 I 7 . I 8 & $-28 \mathrm{I}$ & {$\left[\begin{array}{ll}2 & 4 \mathrm{I} \cdot 3\end{array}\right.$} & 17 & $25 \quad 17.68$ & -28 & I 3 & 30.4 & +0.20 & -0.2 \\
\hline 4 & $a=\mathrm{CoD}-28^{\circ} \mathrm{I} 3234$ & I 7 & $25 \quad 11.09$ & -28 & 2042.3 & 17 & $26 \quad 11.67$ & -28 & $2 \mathrm{I}$ & 30.1 & -0.18 & +0.4 \\
\hline 5 & Gou 23932 & & $34 \quad 37.39$ & $-28 \quad 5$ & $3^{2} \quad 24 \cdot 5$ & 17 & $35 \quad 38.28$ & -28 & 52 & 59.2 & +0.09 & -0.2 \\
\hline 6 & $G Z 17^{\mathrm{h}} 2822$ & I 7 & $43 \quad 54.09$ & $-29 \mathrm{r}$ & $5 \times 3.9$ & 17 & $44 \quad 55.20$ & -29 & I 5 & 35.6 & -0.05 & -0.6 \\
\hline 7 & $\mathrm{GZ} \times 7^{\mathrm{h}} 35^{2}$ & & $\begin{array}{ll}54 & 20.3 \\
I\end{array}$ & -294 & 1942.6 & I 7 & $5.5 \quad 21.73$ & -29 & 49 & 49.8 & -0.10 & -2.5 \\
\hline 8 & Gou 24446 & I 7 & $543^{8.5} \mathrm{I}$ & -294 & 498.6 & 17 & $\begin{array}{ll}55 & 39.95\end{array}$ & -29 & 49 & $15 \cdot 3$ & +0.05 & $-\mathrm{I} . \mathrm{I}$ \\
\hline 9 & Gou 24536 & I 7 & $\begin{array}{lll}57 & 46.8 \mathrm{I}\end{array}$ & -295 & I 46.3 & I 7 & $58 \quad 48.25$ & -29 & $5 \mathrm{I}$ & 48.6 & +0.04 & -0.3 \\
\hline IO & $\mathrm{GZ}$ 1 $8^{\mathrm{h}} 45$ & & $253.8 \mathrm{I}$ & -30 & $9 \quad 40.3$ & & 355.40 & -30 & 9 & $35 \cdot 4$ & +0.02 & $-1 \cdot 3$ \\
\hline I I & Gou & & $34.5^{8}$ & -30 & 023.0 & & 46.08 & -30 & o & I 8.0 & -0.06 & -1.4 \\
\hline 12 & Gou 24870 & & 10 39.88 & -302 & $6 \quad 6.4$ & & I I 4 I. 59 & -30 & 25 & 50.7 & +0.06 & -0.4 \\
\hline 13 & $\mathrm{GZ}$ I $8^{\mathrm{h}}$ I 400 & & $24 \quad 43.03$ & $-30 \quad 5$ & $53 \times 5.7$ & 18 & $2544.9 \mathrm{I}$ & -30 & 52 & 40.4 & -0.05 & -2.5 \\
\hline 14 & $\mathrm{GZ}$ I $8^{\mathrm{h}} \mathrm{I} 45^{\mathrm{I}}$ & I 8 & $25 \quad 50.15$ & -305 & 58 I 9.7 & & $26 \quad 52.06$ & -30 & 57 & 42.9 & $+0.0 \mathrm{I}$ & -1.5 \\
\hline I 5 & $\mathrm{GZ}$ I $8^{\mathrm{h}} 1806$ & & 324 I.r 6 & -305 & $6 \quad 44.1$ & & 3343.01 & -30 & 55 & 57.8 & +0.08 & -2.6 \\
\hline I 6 & GZ $19^{\mathrm{h}} 37^{\circ}$ & & I० 40.63 & $\begin{array}{ll}-31 & 3\end{array}$ & $34 \quad$ I.6 & 19 & I I 42.29 & $-3 x$ & 32 & 23.6 & -0.04 & -3.0 \\
\hline I 7 & $G Z$ I $9{ }^{\mathrm{h}} 386$ & 19 & 10 57.39 & $-3 \mathbf{I} 3$ & 3519.3 & & I I 59.06 & $-3 I$ & 33 & 40.9 & -0.10 & $-2 \cdot 3$ \\
\hline I 8 & $\mathrm{GZ}$ I $9^{\mathrm{h}} \mathrm{1} 6 \times 8$ & & $40 \quad 23.17$ & $\begin{array}{ll}-3 I & 4\end{array}$ & $4^{6} 5.5$ & 19 & $4 \mathrm{I} 24.3^{\mathrm{I}}$ & $-3 I$ & 44 & 39.7 & -0.01 & -0.6 \\
\hline
\end{tabular}

